\title{
Standard Mismatch - the Production of Controlled Small Reflections in Waveguides*
}

\author{
L. Lewin**
}

(April 26, 1968)

\begin{abstract}
The reflection properties of possible suitable structures, for producing small accurately controlled reflections in rectangular waveguide, are examined and a choice made of cylindrical posts for a more detailed study.

A number of sources of error due to ohmic loss, positioning and shape are investigated, and it is shown that both the inductive and capacitive post can meet reasonable specifications, but that the latter is the more suitable on almost all counts. Attention to post size and tilt is necessary, but the accuracies needed lie well within the range achievable by good engineering practice.
\end{abstract}

Key Words: Controlled small reflections; mismatch; reflector posts; waveguide.

\section{List of Principal Symbols}

a rectangular waveguide broad dimension,

$\overline{\mathbf{a}}_{x}, \overline{\mathbf{a}}_{y}$ unit vectors,

$b \quad$ rectangular waveguide narrow dimension,

$d$ post displacement from guide wall,

j $\quad(-1)^{1 / 2}$,

$k$ wave number in free space, $2 \pi / \lambda$,

$k^{\prime} \quad$ wave number in waveguide, $2 \pi / \lambda_{y}$,

$r$ post radius,

$R$ reflection coefficient,

$T$ transmission coefficient,

$x \quad$ series reactance (normalized),

$X$ parallel reactance (normalized),

$Z$ post impedance (normalized),

$\delta, \Delta$ correction factors due to post conductance

(see eqs (4) and (12)),

$\lambda$ free space wavelength,

$\lambda_{g} \quad$ guide wavelength,

$\theta$ post tilt angle, and

$\sigma \quad$ post conductivity.

\section{Introduction}

The aim is to examine structures which can produce an accurately determined "standard" reflection in an otherwise matched rectangular waveguide. The voltage reflection level required is in the range 0.001 to 0.01 . The structures should be simple and reliable to construct, be capable of being accurately calculated, and the uncertainties due to the unavoidable errors in

\footnotetext{
*An invited paper.
}

**Present address: Standard Telecommunications Laboratories, Ltd., London Road, Harlow, Essex, England. assembly should be known within limits which ensure adequate maintenance of the expected reflections.

\section{Possible Structures}

There are, of course, an unlimited number of structures which could be contemplated for the purpose of producing a standard reflection of small magnitude. Almost any slight disturbances to the guide will suffice. Small perturbations can be accurately calculated, and provided they can be reliably made and measured, could form the basis of a standard reflection.

In order to keep this survey within reasonable bounds, a suitable selection from the possible forms must be made. We shall exclude all dielectric inserts on the grounds that, in addition to their geometry, an accurate figure for the dielectric constant needs to be known over a working temperature range. This makes such inserts possibly unsuitable as a standard.

The shapes which lend themselves readily to both calculation and to mechanical construction include inductive and capacitive rods and diaphragms. However, the diaphragm insert, in addition to requiring a correction for its finite thickness, has the complication that it contains two sharp edges. Since the sharpness can never be precisely defined, this makes it difficult to estimate errors arising from departures from a perfect geometrical form. Moreover, a very high current density flows at the sharp edges, making a possible loss contribution to be considered. For these reasons we will leave out the diaphragms from this survey.

We shall examine the inductive and capacitive rods in the next two sections. 


\section{Inductive Rods}

\subsection{The Post Reactance}

A rod of radius $r$ is placed across the guide a distance $d$ from one (narrow) guide wall as shown in figure 1. We are interested in small reflections, and therefore both $d$ and $r$ will be small. The dominant expression for the obstacle reactance $X$, which is connected with the reflection $R$ by $R=-1 /(1+j 2 X)$ is

$$
\begin{aligned}
X= & \frac{a}{2 \lambda_{g}} \operatorname{cosec}^{2}(\pi d / a)\{\log [(2 a / \pi r) \sin (\pi d / a)] \\
-\sin ^{2}(\pi d / a) \cdot\left(2+k^{2} a^{2} / \pi^{2}\right) & +k^{2} d^{2}[-\log (2 \pi d / a) \\
& \left.\left.+3 / 2+\pi^{2} d^{2} / 36 a^{2}\right]\right\}
\end{aligned}
$$

This is eq $(2.16)^{1}$ of the publication cited in footnote 1 and is suitable for use for small $d$, giving large $X$ and hence a small reflection. A more rigorous form is eq (2.14) [1] and a numerical calculation may be necessary in particular cases to confirm that eq (l) is sufficiently accurate.

\subsection{Errors of Positioning}

As an extremely crude, order of magnitude, approximation, eq (1) may be put in the form

$$
X \simeq 1 /(\pi d / a)^{2}
$$

which shows that $R$ is proportional to $d^{2}$. An accurate placing of the rod is therefore necessary. To give $R=0.001$, we need $d \simeq a / 100$. In a 2 -in guide this gives $d=20$ mils; and a 1 -mil error in placing gives rise to a 10 percent error in the reflection. This may seem large, though it is actually only a 0.0001 reflection, which is likely to be of the same order as effects due to other errors in the system. However, it seems undesirable to permit a 10 percent error, and either a more accurate construction is needed or else an

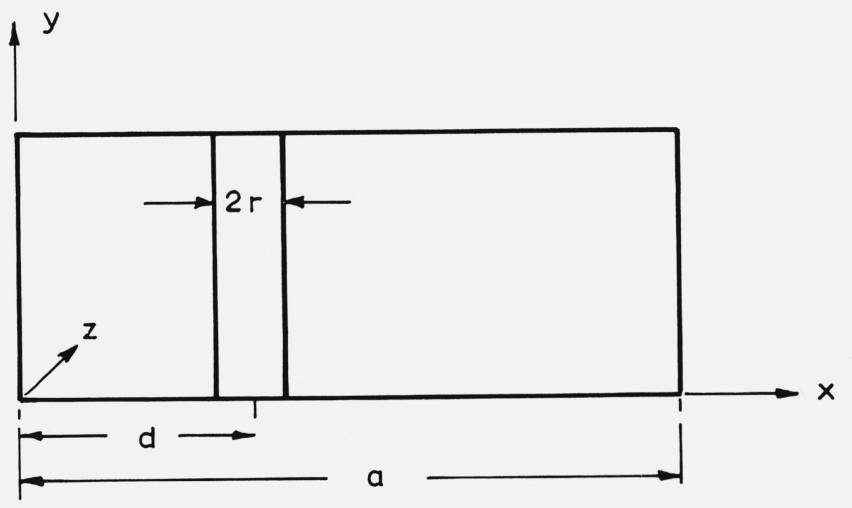

FIGURE 1. Inductive post.

1 Lewin, L., Advanced Theory of Waveguides, pp. 26, 27, 35, 44, and 77 (Iliffe and Sons, Ltd., London, 1951). alternative structure which is less sensitive to position. (Of course, jig-boring to a much better accuracy than 1 mil can be achieved if it is really required.)

When $R=0.01, d$ is about three times larger, and the relative error in reflection is about 3 percent for a 1-mil positional error.

\subsection{Post Tilt}

If the post is set at an angle $\theta$ to the normal, the effective field at the post is reduced by a factor $\cos \theta$, while its effect back into the guide is reduced by the same amount. An estimate of $\theta$ might be, for example, 1 mil in 1 in giving $\theta=10^{-3} \mathrm{rad}$, for which $\cos ^{2} \theta$ differs insignificantly from unity.

In addition to this effect, a cross-component of electric field proportional to $\sin \theta$ will introduce a capacitive post effect, proportional to both $\sin ^{2} \theta$ and the square of the post radius. This term is completely negligible.

\subsection{Finite Post Radius}

A higher-order correction to eq (1) is given by eq (2.43) of footnote 1. For small $r$ and $d$ the fractional correction is, very approximately, $r^{2} / d^{2}$. Thus if $d=20$ mils in a 2 -in guide (to give a 0.001 reflection), the percentage error, if the wire radius is 5 mils, is about 7 percent. For a 0.01 reflection, $d$ in this example is about 60 mils and an error of about 1 percent can be expected. If these errors are too great to be tolerated for a particular application, equation (2.43) should be used in preference to eq (1). It may also be desirable to use a somewhat larger wire diameter, in which case the more accurate formula should definitely be used.

\subsection{Finite Post Resistivity}

In appendix 1 it is shown that the effect of a conductivity $\sigma$ for the post material leads to the normalized impedance of the post, $j X$, being augmented to

$$
Z=(1+j) k^{\prime} a /\left[8 \pi k r \sin ^{2}(\pi d / a)(30 \lambda \sigma)^{1 / 2}\right]+j X
$$

Since $X=0(1) / \sin ^{2}(\pi d / a)$, the fractional correction due to finite conductivity can be estimated from the expression

$$
\Delta=k^{\prime} a / 8 \pi k r(30 \lambda \sigma)^{1 / 2}
$$

As an example to show the order of magnitude, take $r=1 \mathrm{~mm}, a=5 \mathrm{~cm}, \lambda=7.5 \mathrm{~cm}, \sigma=5.10^{5} \mathrm{mho} / \mathrm{cm}$.

Then $\Delta \simeq 10^{-4}$, and the effect is completely negligible. The post radius would have to be quite minute for an effect to show up.

\subsection{Conclusions for Inductive Post}

Of the four possible causes of error considered, the effects of tilt and post resistivity are negligible. The corrected formula (2.43) (see footnote 1) for finite post radius should probably be used, in which case, 
no error should occur from this source. Due to the rapid rise of the reflection as the post moves away from the side wall, a very accurate positioning is needed, but this should be within the bounds of good engineering practice. A possible further source of error can come from the contact where the post meets the guide. If this is soldered, the quality of the contact may not be known for certain, and possible further effects due to the extrusion of solder into the guide may occur. The latter may be avoided by using a technique in which the rods are very slightly oversize, are shrunk in liquid nitrogen, and then allowed to expand in situ. The uncertain effect of the contact resistance remains, however.

It is concluded that the inductive post is a possible but not ideal solution to the problem.

\section{Capacitive Rods}

\subsection{The Post Reactance}

Equations (2.74) and (2.75) (see footnote 1) give the series and parallel components of the equivalent circuit. (Note that $k$ must be replaced by $k^{\prime}$.) The formulas contain correction terms due to the finite post radius. Ignoring these for the moment, we have two equal series arms of normalized reactance magnitude

$$
x=-\pi k^{\prime} r^{2} / 2 b
$$

and a parallel arm of magnitude

$$
X=-b /\left(2 \pi k^{\prime} r^{2}\right)
$$

The reflection and transmission coefficients are given by

$$
\begin{aligned}
& j x=(1+R-T) /(1-R+T) \\
& j\left(X+\frac{1}{2} x\right)=-\frac{1}{2}+1 /(1-T-R)
\end{aligned}
$$

To terms in order $r^{2}$ this gives

$$
R \simeq j[x+1 /(2 X)]=-j 3 \pi k^{\prime} r^{2} / 2 b
$$

For example, if $b=2.5 \mathrm{~cm}, \lambda=7.5 \mathrm{~cm}$, then $r \simeq 1 \mathrm{~mm}$ for a reflection of 0.01 , and corresponding values proportional to $r^{2}$ for different radii.

\subsection{Errors of Positioning}

The formula quoted is for a central post, and therefore cannot be used for estimating positional errors. On general grounds, however, it can be said that if an obstacle is placed at a position $d$ where the relevant mode has a magnitude $\Phi(d)$, then the variation of the reflection with $d$ will involve $\Phi^{2}(d)$ as the dominant effect. This can be seen from eq (1), for example, where the factor $\operatorname{cosec}^{2}(\pi d / a)$ in the reactance (which is inverted for the reflection coefficient) comes from
$\Phi(d)=\sin (\pi d / a)$, the form of the dominant waveguide mode.

In the present case, the dominant mode is unvarying with $d$, since it is constant across the guide. Except for proximity effects when $d$ is very small, there is no other variation. Hence, for a central post (and there seems no point in taking it off center), no errors of positioning need be expected.

\subsection{Errors of Radius}

Since $R \propto r^{2}$, we have, for small errors $\delta r, \delta R$

$$
\delta R / R=2 \delta r / r .
$$

An error of 1 mil in a 25 mil radius post gives a 4 percent reflection error. Since rods can certainly be turned to a much greater accuracy than this, errors due to incorrect post diameters can certainly be made negligible.

\subsection{Finite Post Radius}

The correction terms to eqs (5), (6), and (8) are of relative order $\left(k^{\prime} r\right)^{2}$, or about 1 percent. This is probably negligible, but resort to the exact formulae in any particular case will give the correct reflection.

\subsection{Finite Post Conductivity}

As with the inductive post, the effect on rods of practical size is completely negligible.

\subsection{Post Tilt}

If the post is tilted at an angle $\theta$, as shown in figure 2 , a component of electric field proportional to $\sin \theta$ is set up along the post. This will excite the post as if it were in the inductive position, giving rise to a post current which will reradiate back into the guide by an amount containing a further factor $\sin \theta$. The analysis is given in appendix 2, where it is shown that an approximation to the reflection is

$$
\delta R=\pi k b \sin ^{2} \theta /\left[\left(k^{\prime} b\right)^{2} \log (\pi r / 2 b)\right] .
$$

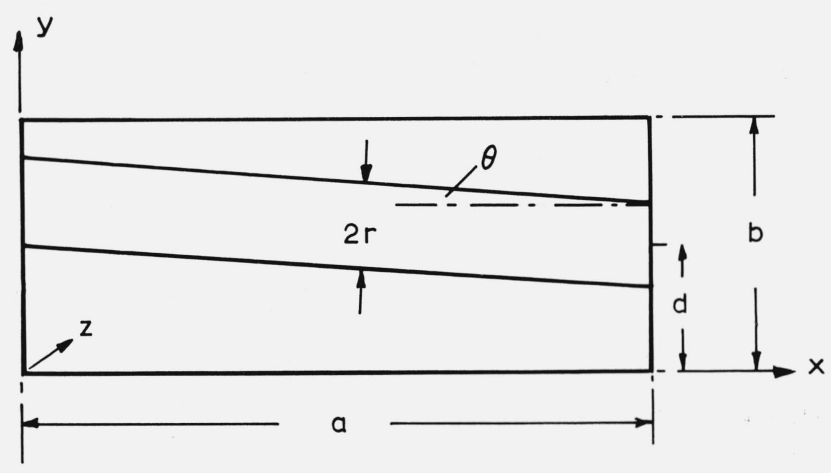

Figure 2. Tilted capacitive post. 
If we take $b=2.5 \mathrm{~cm}, r=1 \mathrm{~mm}, \lambda=7.5 \mathrm{~cm}$, then

$$
\delta R \simeq-\sin ^{2} \theta
$$

An estimate of $\theta$ might be 2 -mils error in 2 in, $\theta=10^{-3}$ radian and $\delta R=-10^{-6}$, and is only 0.1 percent of a reflection 0.001 . We can therefore conclude that the effect of tilt can be made negligible.

\subsection{Conclusions for Capacitive Post}

Of the five possible causes of error, the effects of positioning and finite conductivity are completely negligible, while the effects of tilt, though potentially harmful, can be completely avoided by the achievement of the accuracy accompanying good engineering practice. The second-order corrections to the formulas, as given (see footnote 1), though probably negligible, can be readily calculated and allowed for.

The remaining source of error lies in the possible errors in machining the post diameter to size. The example used a 1-mil error in a 25 -mil radius post, but it should be possible to achieve an order of magnitude better if it is really needed.

A possible source of error due to the post buckling has not been investigated, since this should not occur with a well engineered design. However, the axial (inductive post) effect should cancel to a first order for a symmetrical buckling, while the displacement effect should also be quite negligible.

Since no axial current flows in the capacitive post, the condition of the soldering at the ends seems to be a minor factor. The shrink-on process should be ideal here, though it would be as well to take precautions against buckling during the final expansion.

It is concluded that the capacitive post has all the requirements of a suitable low-reflecting obstacleease of accurate manufacture, absence of effects of positioning or other undesirable geometrical factors, absence of severe contact effects, and a fairly straightforward formula for the reflection. It also has the considerable advantage that undesirable higher-order modes are rapidly attenuated along the guide.

\section{Conclusions}

It is concluded that the central capacitive post is an almost ideal solution to the problem of achieving standard reflections in the 0.01 to 0.001 range, or, for that matter, over a more extended range.

This study was undertaken under the sponsorship of the Radio standards Laboratory of the National Bureau of Standards.

\section{Appendix 1. Effect of Finite Post Conductivity}

The calculation proceeds almost exactly as stated in footnote 1 except that the boundary condition at the post, instead of being $E=0$ becomes

$$
E=I(30 / \lambda \sigma)^{1 / 2}(1+j) / r=I \delta,
$$

(see eq (4.2) see footnote 1).

Instead of (2.10) we get

$$
\begin{aligned}
I=\sin (\pi d / a) / & {[\delta+(60 \pi j k / a)} \\
& \left.\sum_{1}^{\infty} \frac{\cos (m \pi r / a)-\cos (2 m \pi d / a)}{\Gamma_{m}}\right]
\end{aligned}
$$

Accordingly, the post impedance becomes

$$
Z=j X+a \delta k^{\prime} \operatorname{cosec}^{2}(\pi d / a) / 240 \pi k
$$

where $j X$ is the reactance for infinite conductivity. Equation (4) of the text follows directly.

\section{Appendix 2. Effect of Tilt on Capacitive Post}

We assume a capacitive post located across the guide at $y=d$, the guide being excited by a dominant mode of form $E_{0} \sin (\pi x / a) \overline{\mathbf{a}}_{y}$ at the post. If the post is tilted at a small angle $\theta$ from the perpendicular, a component of field will be produced along the post, of magnitude

$$
E_{0} \sin \theta \sin (\pi x / a) \overline{\mathbf{a}} \mathbf{x}
$$

This will cause radiation into the guide much as if the post were in the inductive position. We assume the field sets up a post current

$$
i=I \sin (\pi x / a) \delta(z) \delta(y-d)
$$

where $I$ is a magnitude to be determined. The Hertzian vector has an $x$-component $\Pi_{x}$ satisfying

$$
\nabla^{2} \Pi_{x}+k^{2} \Pi_{x}=(120 \pi j / k) I \sin (\pi x / a) \delta(z) \delta(y-d)
$$

Assume $\Pi_{x}=\Pi \sin (\pi x / a)$ where $\Pi$ is independent of $x$. Since $E=\operatorname{grad} \operatorname{div}\left(\mathbf{a}_{x} \Pi_{x}\right)+k^{2} \mathbf{a}_{x} \Pi_{x}$ we get, for the $x$-component of $E$, the equation $E_{x}=k^{\prime 2} \Pi_{x}$, while $\nabla^{2}+k^{2} \equiv \partial^{2} / \partial y^{2}+\partial^{2} / \partial z^{2}+k^{\prime 2}$. Hence

$$
\begin{aligned}
\left(\partial^{2} / \partial y^{2}\right. & \left.+\partial^{2} / \partial z^{2}+k^{\prime 2}\right) E_{x} \\
& =\left(120 \pi j k^{\prime 2} / k\right) I \delta(z) \delta(y-d) \sin (\pi x / a)
\end{aligned}
$$

The solution to this equation is obtained as in reference [1], p. 25, giving

$$
\begin{aligned}
& E_{x}=\left(-j 120 \pi k^{\prime 2} / k b\right) I \sin (\pi x / a) \\
& \sum_{1}^{\infty} \sin \left(\frac{m \pi d}{b}\right) \sin \left(\frac{m \pi y}{b}\right) \frac{e^{-\Gamma_{m}|z|}}{\Gamma_{m}}
\end{aligned}
$$


where $\Gamma_{m}=\left(m^{2} \pi^{2} / b^{2}-k^{\prime 2}\right)^{1 / 2}$. Equating this to the negative of the exciting field (eq 15) at $y=d+r$ determines $I$ to give zero axial tangential field at the post surface.

$I=\frac{E_{0} \sin \theta k b}{60 \pi j k^{\prime 2}} \sum_{1}^{\infty} \frac{1}{\Gamma_{m}}[\cos (m \pi r / b)-\cos (2 m \pi d / b)]$

This current flows along the post, i.e., in the $x$ direction. Due to the post tilt, a component $I \sin \theta$ reradiates back with polarization in the $y$ direction. The formula for this radiation is given by eq (2.48) (see footnote 1 ), in which the dipole strength $M$ is taken equal to $(-120 \pi j / k b) I \sin \theta$. The dominant mode reradiated back into the guide comes from the first term in the series of modes of eq (2.48), from which the magnitude of the electric field is $(-60 \pi / b) I \sin \theta$. Inserting for $I$ from (20) gives the reflection due to the tilt

$$
\delta R_{\mathrm{tilt}}=\frac{-k \sin ^{2} \theta}{k^{\prime 2} \sum_{1}^{\infty} \frac{1}{\Gamma_{m}}[\cos (m \pi r / b)-\cos (2 m \pi d / b)]}
$$

Taking $d=b / 2$, this formula can be put in the approximate form, suitable for small $r$,

$$
\delta R_{\mathrm{tilt}}=\frac{\pi \sin ^{2} \theta(k b)}{\left(k^{\prime} b\right)^{2} \log (\pi r / 2 b)}
$$

which is eq (10) of the text.

(Paper 72C3-277) 